Mathématiques et sciences humaines
Mathematics and social sciences

135 | Automne 1996

Varia

\title{
Construction d'un schéma d'implication entre variables issues d'une analyse des correspondances multiples. Développements. Application
}

Construction of an involvement scheme among variables issued from a multiple correspondence analysis. Developments. Application

\section{Emile-Henri Riard et Mouloud Tensaout}

\section{OpenEdition \\ Journals}

Édition électronique

URL : http://journals.openedition.org/msh/2721

DOI : $10.4000 /$ msh.2721

ISSN : 1950-6821

Éditeur

Centre d'analyse et de mathématique sociales de l'EHESS

Édition imprimée

Date de publication : 1 septembre 1996

ISSN : 0987-6936

\section{Référence électronique}

Emile-Henri Riard et Mouloud Tensaout, "Construction d'un schéma d'implication entre variables issues d'une analyse des correspondances multiples. Développements. Application », Mathématiques et sciences humaines [En ligne], 135 | Automne 1996, mis en ligne le 10 février 2006, consulté le 23 juillet 2020. URL : http://journals.openedition.org/msh/2721; DOI : https://doi.org/10.4000/msh.2721

Ce document a été généré automatiquement le 23 juillet 2020.

(c) École des hautes études en sciences sociales 


\section{Construction d'un schéma} d'implication entre variables issues d'une analyse des correspondances multiples. Développements. Application

Construction of an involvement scheme among variables issued from a multiple correspondence analysis. Developments. Application

\section{Emile-Henri Riard et Mouloud Tensaout}

\section{RÉSUMÉS}

L'une des limites de l'analyse des correspondances multiples appliquée à de grands tableaux de données qualitatives est la difficulté d'analyse et d'interprétation des structures de relations entre variables. Afin de dépasser la frontière descriptive, il est proposé une méthodologie de recherche de schémas d'implication reposant sur les fréquences conditionnelles données par les tableaux de Burt. L'analyse des correspondances multiples y est utilisée comme filtre principal de variables à partir desquelles, et dans le cadre des méthodes explicatives, sont construits des graphes orientés. Ils sont accompagnés d'indices (fonction, direction d'influence, force motrice, outre celui de masse) qui apportent une plus grande lisibilité des variables impliquées et ouvrent sur d'autres analyses statistiques avancées.

One of the limits of multiple correspondence analysis applied on large tables of qualitative items, is the difficulty of analysis and interpretation on the structure of relations among variables. In 
order to outpass the descriptive frontier, we propose a research methodology on involvement schemes which is based on conditional frequencies given by Burt's tables. The multiple correspondence analysis is used as a principal filter for variables from which (and as part of explanatory methods) oriented graphs are buit-up. They are accompagnied by some indicators (function, influence direction, driving force, besides one of the mass), which provide a greater legibility of the variables involved, and open the way to other advanced statistical analysis.

\section{INDEX}

Thèmes : données (analyse des), inférence, méthodologie, statistique 